\title{
Validade do conjunto de testes da habilidade de memória de curto-prazo (CTMC)
}

\author{
Cristiano Mauro Assis Gomes \\ Universidade Federal de Minas Gerais
}

\begin{abstract}
Resumo
Este artigo analisa em nível exploratório a validade do CTMC, em termos de sua estrutura fatorial, validade convergente, validade discriminante e validade preditiva. Serão analisadas as respostas de 230 participantes que cursavam o ensino médio. Para investigar a estrutura fatorial foi usada a análise fatorial exploratória. Para investigar a validade convergente, divergente e preditiva foi usado o modelamento por equação estrutural. Os resultados apontam que os escores dos testes possuem adequada confiabilidade e validade. A estrutura fatorial dos itens dos testes indica a presença de três fatores especializados da memória de curto-prazo (Gsm). Os testes convergem para mensurar Gsm e divergem na mensuração de Gf. Gsm, medida pelo CTMC, prediz Gf.

Palavras-Chave: memória de curto prazo; validade; inteligência.
\end{abstract}

\begin{abstract}
Short term memory ability tests kit validity (CTMC). This article is an exploratory study of CTMC validity. It investigates the CTMC factorial structure, and the convergent, divergent and predictive validity. Exploratory factor analysis was used to evaluate the factor structure of CTMC from 230 high school students' data. Structural equation modeling was used to evaluate the convergent, divergent and predictive validity. The result shows the reliability and validity of tests scores. The factorial structure of test items indicates three specialized factors of the short term memory (Gsm). Tests converge to measure Gsm while diverge to measure Gf. Gsm measured by CTMC predicts Gf.

Keywords: short term memory ability; validity; intelligence.
\end{abstract}

\section{A mensuração de habilidades cognitivas de alta- ordem}

Carroll (1993) realizou uma meta-análise gigantesca sobre os estudos psicométricos do campo da inteligência. Ele coletou cerca de 1500 matrizes de correlação obtidas no século XX e analisou cerca de 461 dessas matrizes. Seus resultados ajudaram a solidificar as evidências de que a inteligência é composta por níveis ou estratos. O primeiro estrato é composto por dezenas de habilidades cognitivas específicas e dizem respeito a processos cognitivos especializados relacionados a um domínio psicológico bem definido, como é o caso da habilidade de raciocínio dedutivo, a habilidade de rotação mental de objetos, a habilidade de memória associativa, etc. O segundo estrato é composto por um número bem menor de habilidades cognitivas. Na época, Carroll (1993) encontrou oito habilidades nesse estrato e as definiu como processos cognitivos amplos, não tão especializados como as habilidades do primeiro estrato, mas circunscritos a um domínio psicológico bem definido. Dentre essas habilidades encontram-se, por exemplo, a inteligência fluida, a inteligência cristalizada e a memória de curto-prazo. O terceiro estrato é composto exclusivamente por uma única habilidade, o fator geral de inteligência. Com relação aos estratos, Carroll (1993) chamou as habilidades do estrato dois e três de habilidades de alta-ordem, na medida em que elas são identificadas a partir da correlação das habilidades de primeira ordem.

Em função dos avanços nos modelos psicométricos a partir do estudo de Carroll (1993), houve uma mudança importante na geração e alteração das baterias de inteligência. Se nos anos de 1990 havia uma carência razoável de baterias capazes de mensurar um grande número de habilidades cognitivas de alta-ordem (Carroll, 2003; Roberts et al., 2000; Tirre \& Field, 2002; Watkins, Wilson, Kotz, Carbone, \& Babula, 2006), essa situação se inverteu posteriormente e um conjunto significativo de baterias internacionais passou a incorporar várias habilidades cognitivas de alta-ordem em sua medida. Segundo Alfonso, Flanagan e Radwan (2005), até 1998 as principais baterias existentes mensuravam de duas a três habilidades de alta-ordem (excetuando o fator geral). Após 1998, essas mesmas baterias passaram a mensurar cerca de quatro a cinco habilidades de altaordem (sem contar com $g$ ). Por outro lado, apesar dos avanços, deve-se reconhecer que nenhuma bateria atual mensura todas as habilidades cognitivas de alta-ordem. 
Alguns estudos no Brasil que avançam na elaboração de baterias para a mensuração de habilidades de alta-ordem podem ser destacados, entre eles a validação do WJIII (Wechsler \& Schelini, 2006), a adaptação brasileira dos testes verbais do WJIII (Wechsler, Vendramini, \& Schelini, 2007), a bateria multidimensional para a inteligência infantil (Schelini \& Wechsler, 2005, 2006), e a Bateria de Fatores Cognitivos de Alta-Ordem (BaFaCAlO). Esta é menos conhecida e foi elaborada por Gomes (2010) para medir, em amostras brasileiras, o fator geral $(g)$ de terceiro nível do modelo de Carroll (1993), assim como seis das oito habilidades cognitivas de segundo nível deste modelo: a Inteligência Fluida (Gf), a Inteligência Cristalizada (Gc), a Habilidade Visuo-Espacial (Gv), a Fluência (Gr), a Memória de Curto-Prazo (Gsm) e a Rapidez Cognitiva (Gs). A BaFaCAlO é formada por 18 testes de inteligência, do tipo lápis e papel, com um tempo limite para sua resolução. Em termos procedimentais, a bateria é aplicada individual ou coletivamente, por psicólogos ou estudantes de psicologia devidamente treinados. Os 18 testes formam seis conjuntos de testes: Conjunto de Testes de Inteligência Cristalizada (CTIC), Conjunto de Testes de Inteligência Fluida (CTIF), Conjunto de Testes de Habilidade Visuo-Espacial (CTVE), Conjunto de Testes da Habilidade de Memória de Curto Prazo (CTMC), Conjunto de Testes de Fluência (CTF), e o Conjunto de Testes de Rapidez Cognitiva (CTRC).

A BaFaCAlO foi criada em meados dos anos 2000 e por isso há apenas três estudos publicados sobre a bateria. Eles apresentam evidências que apóiam sua validade de construto. Gomes (2010) analisou a estrutura fatorial da BaFaCAlO e comparou via modelagem por equação estrutural o ajuste de três soluções fatoriais exploratórias, obtidas por diferentes técnicas de retenção de fatores. O modelo de melhor ajuste foi o que postulava seis fatores de primeiro nível, Gf, Gc, Gr, Gs, Gsm e Gv, e um fator de segundo nível, representando $g\left(\chi^{2}=\right.$ $169,67 ; g l=122 ; \mathrm{CFI}=0,98 ; \mathrm{RMSEA}=0,04)$. Concomitante, os testes da $\mathrm{BaFaCAlO}$ foram explicados pelas suas habilidades cognitivas alvo, com uma carga fatorial igual ou superior a 0,30 . Os 18 testes apresentaram um alfa de Cronbach acima de 0,70.

Analisando separadamente os conjuntos da BaFaCAlO, Gomes e Borges (2009a) investigaram, em uma amostra de 558 estudantes de ensino médio, a estrutura fatorial dos instrumentos do Conjunto de Testes de Inteligência Fluida (CTIF), a unidimensionalidade de cada teste, assim como a validade convergente e discriminante do Conjunto. Os resultados indicaram que cada teste do CTIF apresentou uma estrutura fatorial complexa, embora sua medida tenha bom ajuste ao modelo unidimensional Rasch. Os testes do CTIF apresentaram quantidade equilibrada de itens fáceis, medianos e difíceis, e são explicados (convergem) por uma mesma habilidade cognitiva, Gf, com cargas fatoriais acima de 0,70 . Concomitante, eles não são explicados (divergem) por outra habilidade cognitiva, Gr, que explica três testes de fluência. Além do CTIF, Gomes e Borges (2009b) investigaram a unidimensionalidade da medida e a estrutura fatorial dos testes do CTVE, sua validade convergente e validade divergente. Os resultados indicam que os testes possuem uma estrutura fatorial complexa, mas uma medida com bom ajuste ao modelo unidimensional Rasch, convergindo para a mensuração da habilidade-alvo, Gv, com uma carga fatorial acima de 0,64, e divergindo da medida de Gr.

\section{O conjunto de testes da habilidade de memória de curto prazo (ctmc)}

A habilidade de memória de curto prazo (Gsm) é uma habilidade do estrato dois do Modelo de Três Estratos de Carroll (1993). Ela é concebida pela psicometria como uma habilidade de alta-ordem (Bickley, Keith, \& Wolfle, 1995; Carroll, 1993; Cole \& Randall, 2003; Johnson \& Bouchard Jr., 2005a, 2005b; Johnson, te Nijenhuis, \& Bouchard Jr., 2007; Marañon \& Andrés-Pueyo, 2000; Undheim \& Gustafsson, 1987). Enquanto habilidade de alta-ordem, Gsm representa um processo cognitivo genérico, o qual predomina um determinado processamento, conteúdo ou domínio psicológico. Descrevendo-a, Gsm indica situações, tarefas, desafios, problemas, etc, os quais há o predomínio do processamento de ativação e manutenção de determinados estímulos durante um curto período de tempo (McGrew \& Flanagan, 1998; Primi, 2003). Gsm é mensurada por habilidades cognitivas do estrato um, como é o caso da memória associativa (MA), da memória de retenção (MS) ou da memória visual (MV) (Carroll, 1993; Spearrit, 1996) e seus testes marcadores são instrumentos que mensuram estas habilidades.

Gsm tem interessado a vários pesquisadores do campo psicométrico. Um dos motivos desse interesse refere-se ao fato de que as habilidades de alta-ordem, de uma maneira geral, apresentam bom poder preditivo em relação a uma série de desempenhos humanos, como o rendimento escolar e a performance profissional (Capovilla \& Capovilla, 2001; McGrew, Keith, Flanagan, \& Vanderwood, 1997; Rueda, Sisto, Cunha, \& Machado, 2007; Rueda, Sisto, Cunha, Machado, Moraes Júnior et al., 2007; Spearrit, 1996).

O Conjunto de Testes da Habilidade de Memória de Curto Prazo (CTMC) é um conjunto de instrumentos de medida da $\mathrm{BaFaCAlO}$. Ele tem como objetivo a medida de Gsm e, para isso, possui três testes: (1) Teste de Memória Associativa 1 (MA1), (2) Teste de Memória Associativa 2 (MA2), e (3) Teste de Memória Visual (MV). Eles foram construídos a partir da referência da estrutura dos testes de memória de curto prazo do Kit of Factor-Referenced Cognitive Tests (Ekstrom, French, Harman, \& Dirmen, 1976).

O Teste de Memória Associativa 1 (MA1) é composto por 15 itens. Ele possui uma folha de memorização e uma folha de resposta. Na folha de memorização o respondente deve memorizar a relação entre um objeto e um número de dois dígitos. A folha de resposta apresenta, em ordem diferente da folha de memorização, todos os objetos sem o seu número correspondente, de modo que o respondente deve escrever o número relacionado a cada objeto. O Teste de Memória Associativa 2 (MA2) é composto por 15 itens. Possui uma folha de memorização e uma folha de resposta. $\mathrm{Na}$ folha de memorização o respondente deve memorizar a relação entre o nome e o sobrenome de 15 pessoas. A folha de resposta apresenta, em ordem diferente da folha de memorização, todos os sobrenomes sem a presença do nome correspondente, de modo que o respondente deve escrever o nome relacionado a cada sobrenome. O Teste de Memória Visual (MV) possui 12 itens, uma folha de memorização e uma folha de resposta. A 
folha de memorização apresenta 12 mapas. A folha de resposta apresenta também 12 mapas e duas opções de resposta, onde o respondente deve indicar se o mapa da folha de resposta é exatamente o mesmo da folha de memorização.

Buscando mensurar a memória de curto prazo (Gsm), o CTMC é composto por testes que são marcadores de duas habilidades especializadas ou, como queira, habilidades de primeiro estrato do modelo de Carroll (1993): memória associativa (MA) e memória visual (MV). Superficialmente, essa escolha pode parecer inadequada, na medida em que testes marcadores de Gsm deveriam ser elaborados para medir exclusivamente esta habilidade de alto nível e não habilidades especializadas ou do primeiro estrato. Essa aparentemente inadequação não retrata genuinamente a situação. Para explicá-la, é preciso informar que o modelo teórico e empírico que sustenta o Conjunto de Testes de Memória de Curto Prazo é o modelo dos três níveis de Carroll (1993). As evidências de Carroll (1993) mostram que qualquer teste de inteligência, situado dentro das atuais tecnologias de construção de instrumentos, mensura obrigatoriamente pelo menos três habilidades cognitivas, situadas em três níveis hierárquicos distintos. Carroll (1993) evidencia que qualquer teste de inteligência mede o fator geral de inteligência. Ele explica que esta habilidade cognitiva se situa em um nível altamente geral de processamento cognitivo e chama essa camada de terceiro estrato ou nível três. Além da relação obrigatória com o fator geral de inteligência, os resultados de Carroll (1993) indicam que todo teste de inteligência também mensura uma habilidade cognitiva de caráter amplo, mas não tão geral quanto $g$. Conforme já argumentado, ele obteve dados empíricos de que habilidades dessa complexidade de processamento fazem parte de uma camada que denominou de segundo estrato ou nível dois. Carroll (1993) também verificou que qualquer teste de inteligência mede uma habilidade específica, relacionada a processos cognitivos mais especializados. Recapitulando o que já foi dito, ele obteve evidências de que todas as habilidades específicas desse tipo se situam em uma camada denominada de primeiro estrato ou nível um.

Através de seu modelo de habilidades situadas em níveis hierárquicos, Carroll (1993) identificou que a memória de curto prazo (Gsm), uma habilidade de segundo nível ou estrato, é relacionada diretamente a cinco habilidades especializadas de primeiro nível (estrato): memória de retenção de ordem, memória associativa, memória significativa, memória sem significado e memória visual. Situando os testes do CTMC ao modelo de Carroll (1993), eles foram elaborados de forma a medir conjuntamente (1) o fator geral de inteligência e (2) Gsm. Por sua vez, o Teste de Memória Associativa 1 (MA1) e o Teste de Memória Associativa 2 (MA2) foram elaborados para também mensurar a habilidade de memória associativa (MA) e o Teste de Memória Visual foi elaborado para medir, além de $g$ e Gsm, a habilidade de memória visual (MV), ambas habilidades cognitivas do primeiro estrato. Em síntese, o modelo hipotetizado de medida do Conjunto de Testes de Memória de Curto-Prazo é o seguinte: Os testes MA1 e MA2 devem ser explicados por $g$, Gsm e MA. O Teste MV deve ser explicado por $g$, Gsm e MV.

Os testes do CTMC possuem conteúdo válido para a mensuração das habilidades-alvo de primeiro estrato. $\mathrm{O}$ teste de memória visual possui como estímulos mapas que devem ser memorizados e correspondem aos argumentos de Beier e Ackerman (2004), que explicam que a memória visual tende a ser mensurada adequadamente por tarefas que apresentam estímulos visuo-espaciais, principalmente formas não comuns ou mapas. Os testes de memória associativa do CTMC também possuem conteúdo válido. Beier e Ackerman (2004) mostram que a memória associativa tende a ser bem mensurada por pares de estímulos onde um membro do par permanece sempre à vista do examinando, enquanto o outro deve ser lembrado. Os testes de memória associativa do CTMC possuem essa característica. A respeito da presença de dois testes de memória associativa no CTMC, Beier e Ackerman (2004) argumentam que o conteúdo dos testes de memória associativa é relevante e que estudos fatoriais tendem a identificar que a memória associativa se distingue em diferentes tipos, como a memória associativa verbal, a memória associativa numérica e a memória associativa espacial, sendo adequada a presença de testes distintos para a mensuração de diferentes tipos de memória associativa.

\section{Validade de preditiva de Gsm mensurada pelo Ctmc}

A memória de curto prazo (Gsm) possui associações com algumas variáveis cognitivas e educacionais (Beier \& Ackerman, 2004; Oberauer, Süß, Wilhelm \& Wittman, 2003). Essas associações indicam que a habilidade da memória de curto prazo tem função preditiva. Entre elas, pode-se citar a relação entre Gsm e o rendimento escolar. Santos e Primi (2005) estipulam que limitações na manutenção e no uso de unidades de informação em um prazo de tempo pequeno provavelmente acarretam dificuldades escolares em tarefas que requerem recordações de informações recém-adquiridas. Santos e Primi (2005) encontram correlações entre testes marcadores de Gsm e dificuldades específicas de aprendizagem de estudantes do ensino fundamental relatadas por seus professores. Seus resultados apontam que a memória de curto prazo visual apresenta uma associação com problemas na capacidade de escrever adequadamente as letras e números, além de dificuldades em cálculo. A memória de curto prazo auditiva apresenta uma associação com dificuldades de leitura. Rohde e Thompson (2007), por sua vez, verificam associações entre a memória de curto-prazo e indicadores do desempenho acadêmico de estudantes entre 18 e 23 anos, controlando a influência de outras habilidades cognitivas, como o fator geral de inteligência.

Outra associação relevante refere-se ao papel de Gsm na explicação da arquitetura cognitiva. Demetriou, Christou, Spanoudis e Platsidou (2002) defendem o argumento de que Gsm é uma habilidade do sistema de processamento da informação, que constrange e oferece suporte para o funcionamento das outras habilidades cognitivas. Segundo os autores, Gsm deve ser entendida como uma habilidade de base que sustenta o funcionamento das outras habilidades. Eles estipulam um modelo hierárquico em nível de cascata, no qual as habilidades são dispostas em termos de constrangimento em relação às outras. Os resultados da pesquisa de Demetriou et al. (2002) trazem evidências favoráveis ao seu modelo. Nesse sentido, Gsm explicaria a variância de outras habilidades cognitivas do estrato dois, como a inteligência fluida e a inteligência cristalizada. 


\section{Objetivos do estudo}

Quanto aos objetivos do presente estudo, a validade do Conjunto de Testes da Habilidade de Memória de Curto prazo será analisada em nível exploratório, em termos da estrutura fatorial, validade convergente, validade discriminante e validade preditiva. A análise da estrutura fatorial busca identificar se somente as habilidades especializadas do primeiro estrato de Carroll (1993) e relacionadas com a memória de curto prazo explicam os itens do CTMC, ou se há outros fatores envolvidos. Por sua vez, a investigação da validade convergente e divergente pretende avaliar se os testes do CTMC convergem para a mensuração de um mesmo traço latente e divergem na mensuração de outro traço latente. A validade preditiva envolve o papel de Gsm como habilidade base da arquitetura cognitiva, segundo o argumento de Demetriou et al. (2002) e será estudada por meio da análise da relação entre Gsm e Gf.

\section{Método}

\section{Participantes}

Serão analisados os dados de 230 estudantes de uma escola federal de ensino médio de Belo Horizonte, Minas Gerais. Do total, $46,50 \%$ dos participantes são do sexo feminino. A idade varia entre 14 e 20 anos, apesar da presença de 21 casos nos quais não foi possível a obtenção da idade, sendo que $11,70 \%$ têm 14 anos, 30,40\% possuem 15 anos, 28,70\% têm 16 anos, 15,20\% têm 17 anos, 2,60\% possuem 18 anos, 1,30\% possuem 19 anos e $0,90 \%$ têm 20 anos. Três $(1,30 \%)$ pais dos estudantes têm o ensino fundamental incompleto, $58(25,20 \%)$ completaram o ensino fundamental, $101(43,90 \%)$ completaram o ensino médio e $65(28,30 \%)$ o ensino superior. Foram três os casos nos quais não se pôde obter a escolaridade do pai. Três (1,30\%) mães dos estudantes têm o ensino fundamental incompleto, 47 (20,40\%) o ensino fundamental completo, $110(47,80 \%)$ possuem o ensino médio completo, $68(29,60 \%)$ têm o ensino superior e dois casos não foram relatados. A renda familiar de $100(43,50 \%)$ estudantes é inferior a cinco salários mínimos, 92 (40,00\%) estudantes relatam renda familiar entre 5 e 10 salários mínimos, $36(15,70 \%)$ relatam renda acima de 10 salários mínimos e dois não relatam renda familiar.

\section{Instrumentos}

O Teste de Memória Associativa 1 (MA1), o Teste de Memória Associativa 2 (MA2) e o Teste de Memória Visual (MV) foram relatados na seção de introdução. Eles definem o Conjunto de Testes da Habilidade de Memória de Curto-Prazo e foram elaborados para medir $g$, Gsm e duas habilidades especializadas de memória de curto prazo: a memória associativa e a memória visual.

Além do CTMC, fazem parte dos instrumentos da pesquisa o Conjunto de Testes de Inteligência Fluida (CTIF), pertencente à $\mathrm{BaFaCAlO}$. Seus testes são:

Teste de Indução (I). Composto por 15 itens e um tempo limite de 14 minutos para sua execução. Cada item é composto por cinco grupos de quatro letras. Entre os cinco grupos há quatro grupos que apresentam um mesmo padrão, uma mesma regra de organização de suas letras. O respondente deve identificar o grupo que não apresenta esse padrão e marcá-lo com um x.

Teste de Raciocínio Lógico (RL). Composto por 30 itens e um tempo limite de 24 minutos para sua execução. Cada item é formado por uma conclusão proveniente de duas premissas lógicas que não têm nenhuma relação com o mundo. O objetivo do respondente é indicar se a conclusão presente no item é adequada ou inadequada em relação às premissas do item. $\mathrm{O}$ respondente deve marcar uma de duas opções dadas.

Teste de Raciocínio Geral (RG). Possui 15 itens e um tempo limite de 18 minutos para sua execução. Cada item é formado por um problema lógico-matemático, composto por um enunciado e um espaço para sua resolução. O respondente deve interpretar o enunciado, resolver o problema e escolher uma das cinco opções de respostas do conjunto de múltiplas-escolhas.

As evidências a respeito da validade e confiabilidade do CTIF foram apresentadas na seção de introdução.

\section{Coleta e análise de dados}

Os estudantes desta amostra fizeram todos os testes da $\mathrm{BaFaCAlO}$. Os testes foram aplicados na própria sala de aula dos estudantes, de forma coletiva. Os testes do CTIF e do CTMC demandaram cerca de dois encontros de 100 minutos. A aplicação foi realizada sempre por psicólogos ou estudantes de psicologia devidamente treinados. Foram tomados todos os cuidados éticos em relação aos participantes e a pesquisa contou com a aprovação de Comitê de Ética da Universidade Federal de Minas Gerais, n. 181/06.

Os escores foram formados da seguinte maneira. Zero para o item com resposta incorreta e um para o item com resposta correta. Os itens não feitos foram pontuados como zero, na medida em que o tempo disponibilizado aos participantes foi o suficiente para que eles fizessem todos os itens dos instrumentos.

Para a identificação da estrutura fatorial dos itens do CTMC foi realizada uma análise fatorial exploratória (AFE), com o método de extração de máxima verossimilhança e matriz tetracórica. Na medida em que a AFE tende a gerar diferentes soluções fatoriais, em função de diferentes critérios de extração e retenção de fatores (Beauducel, 2001; Frazier \& Youngstrom, 2007; Guadagnoli \& Velicer, 1988; Henson \& Roberts, 2006), os índices CFI e RMSEA foram utilizados para verificação da solução com o menor número de fatores com bom grau de ajuste aos dados. Foram consideradas, neste estudo, soluções com ajuste adequado àquelas com CFI igual ou acima a 0,95 e RMSEA igual ou inferior a 0,05. Ambos são valores mais rigorosos, indicadores de bom ajuste (Byrne, 2001). Para a análise, foi utilizado o software Mplus 5.2, que disponibiliza os índices estatísticos descritos para análise das soluções exploratórias geradas.

A investigação sobre a validade convergente, a validade divergente e a validade preditiva foi realizada através do modelamento por equação estrutural. O software Amos 16 foi utilizado para esse propósito. Para a medida dos traços latentes presentes no modelamento estrutural, foram utilizadas as seguintes variáveis. Os testes MA1, MA2 e MV foram marcadores de Gsm. Os testes RL, I e RG foram marcadores 
de Gf. Tanto Gf quanto Gsm são mensurados por três variáveis observáveis. Para que a porção isolada da medida da variável latente Gf (Gf e seus testes marcadores, RL, RG e I) e a porção isolada da variável Gsm (Gsm e MA1, MA2, e MV) não tivessem zero graus de liberdade, as variâncias do erro de MA1 e de MA2 foram definidas como variâncias iguais, assim como a variância do erro de I e a variância do erro de RG foram definidas como iguais entre si. Dessa forma, a porção da medida da variável Gf e a porção da medida da variável Gsm passaram a ter um grau de liberdade, de modo que o ajuste da medida dessas porções pôde ser analisado (Byrne, 2001).

\section{Resultados e discussão}

Todos os testes mostraram-se confiáveis, em termos da consistência interna de seus itens. O teste MA1 apresentou um alfa de 0,85, MA2 obteve um alfa de 0,79 e MV um alfa de 0,77. Ao realizar a análise fatorial exploratória (AFE) de todos os itens do CTMC, o objetivo foi verificar se as habilidades do primeiro estrato de Carroll (1993), memória associativa e memória visual seriam encontradas, ou se outros componentes poderiam ser identificados. Conforme já argumentado, o CTMC foi elaborado para mensurar MA e MV, além de Gsm e $g$. AAFE foi feita através do critério de buscar definir o menor número de fatores necessários capazes de explicar a variância das respostas aos itens, desde que atingido os valores pré-definidos de um CFI $\geq 0,95$ e $\mathrm{RMSEA} \leq 0,05$.

A solução com três fatores atendeu esses critérios (CFI $=0,96$, RMSEA $\left.=0,05, \chi^{2}=1096,62, g l=738\right)$. A Tabela 1 apresenta a estrutura fatorial obtida. Pode-se verificar que o fator 1, por concentrar os itens do teste MA, representa a habilidade de memória associativa numérica. $\mathrm{O}$ fator 2 concentra os itens do teste MA2 e representa, dessa forma, a habilidade de memória associativa verbal. $\mathrm{O}$ fator 3 concentra os itens do teste MV e representa a habilidade de memória visual. Os três fatores identificados se correlacionam bem entre si, de modo a apontar um fator genérico de segunda-ordem. As correlações variam entre 0,30 e 0,52 . Essa é uma evidência favorável à presença de Gsm.

A validade convergente, a validade divergente e a validade preditiva foram investigadas através do modelamento por equação estrutural. Dois modelos foram comparados, de forma a analisar qual deles poderia se ajustar melhor aos dados e oferecer evidências para a análise dos três tipos de validade descritos. O modelo um foi desenhado de forma a postular que os três testes do CTMC são explicados por Gsm e os testes do Conjunto de Testes de Inteligência Fluida são explicados por Gf. Gsm e Gf são explicados pelo fator geral de inteligência. Esse modelo corresponde à teoria usual psicométrica. $\mathrm{O}$ modelo dois foi desenhado de forma a postular as mesmas relações do modelo um entre os testes do CTMC e Gsm e os testes de inteligência fluida e Gf. Diferentemente do modelo um, o modelo dois postula que Gsm é uma habilidade cognitiva de base que sustenta o funcionamento de Gf, de forma que Gsm explica Gf. $\mathrm{O}$ modelo dois corresponde às teorias usuais do processamento da informação e da psicologia cognitiva (Demetriou et al., 2002).

O modelo um $\left(\chi^{2}=20,01, g l=11, \mathrm{CFI}=0,97\right.$; RMSEA
$=0,06)$ apresentou um CFI adequado, acima de 0,95 , mas um RMSEA inadequado de 0,06 , maior do que o critério de corte de 0,05 . Por sua vez, o modelo dois $\left(\chi^{2}=15,96, g l=10, \mathrm{CFI}=0,98\right.$, RMSEA $=0,05)$ apresentou um CFI e um RMSEA adequado, dentro dos critérios de corte que indicam um bom ajuste aos dados. Os dois modelos em questão são nested models (ver Kranzler, Keith, \& Flanagan, 2000), de forma que é possível compará-los subtraindo seus qui-quadrados e graus de liberdade. Comparando-os, tem-se que a diferença entre seus qui-quadrados e graus de liberdade (4,05 qui-quadrados e 1 grau de liberdade de diferença) é estatisticamente significativa $(p=0,04)$, indicando

Tabela 1:

Estrutura fatorial dos itens do CTMC

\begin{tabular}{|c|c|c|c|}
\hline & f1 & $\mathrm{f} 2$ & $\mathrm{f} 3$ \\
\hline mali01 & 0,61 & 0,31 & 0,22 \\
\hline mali02 & 0,77 & 0,32 & 0,40 \\
\hline mali03 & 0,70 & 0,45 & 0,25 \\
\hline mali04 & 0,67 & 0,40 & 0,38 \\
\hline mali05 & 0,84 & 0,47 & 0,23 \\
\hline mali06 & 0,71 & 0,56 & 0,41 \\
\hline mali07 & 0,70 & 0,36 & 0,13 \\
\hline mali08 & 0,43 & 0,19 & 0,11 \\
\hline mali09 & 0,60 & 0,40 & 0,33 \\
\hline mali10 & 0,83 & 0,48 & 0,37 \\
\hline mali11 & 0,64 & 0,32 & 0,50 \\
\hline mali12 & 0,55 & 0,29 & 0,09 \\
\hline mali13 & 0,53 & 0,45 & 0,16 \\
\hline mali14 & 0,75 & 0,38 & 0,19 \\
\hline mali15 & 0,59 & 0,31 & 0,32 \\
\hline ma2i01 & 0,41 & 0,40 & 0,22 \\
\hline ma2i02 & 0,36 & 0,42 & 0,45 \\
\hline ma2i03 & 0,38 & 0,62 & 0,27 \\
\hline ma2i04 & 0,34 & 0,69 & 0,15 \\
\hline ma2i05 & 0,38 & 0,57 & 0,15 \\
\hline ma2i06 & 0,22 & 0,45 & 0,19 \\
\hline ma2i07 & 0,38 & 0,50 & 0,24 \\
\hline ma2i08 & 0,26 & 0,69 & 0,20 \\
\hline ma2i09 & 0,37 & 0,56 & 0,45 \\
\hline ma2i10 & 0,31 & 0,58 & 0,31 \\
\hline ma2i11 & 0,49 & 0,53 & 0,38 \\
\hline ma2i12 & 0,33 & 0,66 & 0,10 \\
\hline ma2i13 & 0,45 & 0,59 & 0,16 \\
\hline ma2i14 & 0,44 & 0,53 & 0,20 \\
\hline ma2i15 & 0,39 & 0,59 & 0,53 \\
\hline mvi01 & 0,36 & 0,44 & 0,52 \\
\hline mvi02 & 0,40 & 0,40 & 0,87 \\
\hline mvi03 & 0,18 & 0,10 & 0,58 \\
\hline mvi04 & 0,29 & 0,26 & 0,90 \\
\hline mvi05 & 0,27 & 0,26 & 0,83 \\
\hline mvi06 & 0,18 & $-0,06$ & 0,27 \\
\hline mvi07 & 0,18 & 0,17 & 0,87 \\
\hline mvi08 & $-0,02$ & 0,02 & 0,26 \\
\hline mvi09 & 0,22 & 0,06 & 0,56 \\
\hline mvi10 & 0,12 & 0,40 & 0,35 \\
\hline mvil1 & 0,16 & 0,22 & 0,84 \\
\hline mvi12 & 0,25 & 0,41 & 0,82 \\
\hline
\end{tabular}


que o modelo dois possui melhor ajuste que o modelo um. A Tabela 2 apresenta os pesos não padronizados das regressões do modelo dois, assim como os erros padrões, a razão crítica e a significância estatística das regressões. Todas as regressões são estatisticamente significativas.

São observados indícios favoráveis à validade convergente e à validade divergente através dos dados fornecidos pela Figura 1. Os testes do CTMC convergem para medir a habilidadealvo, supostamente Gsm. Por sua vez, eles não colaboram diretamente na medida de outra habilidade-alvo, supostamente Gf. Concomitantemente, as cargas fatoriais da habilidade-alvo nos testes do CTMC são satisfatórias, variando entre 0,43 e 0,78.

Há também indícios favoráveis sobre a validade preditiva de Gsm medida pelo CTMC. Através da Figura 1, pode-se observar que Gsm tem uma função explicativa sobre Gf. O modelo dois postula que Gsm é uma habilidade base que constrange o funcionamento de Gf, corroborando os argumentos de Demetriou et al. (2002). Neste modelo, Gsm explica 21\% da variância de Gf.

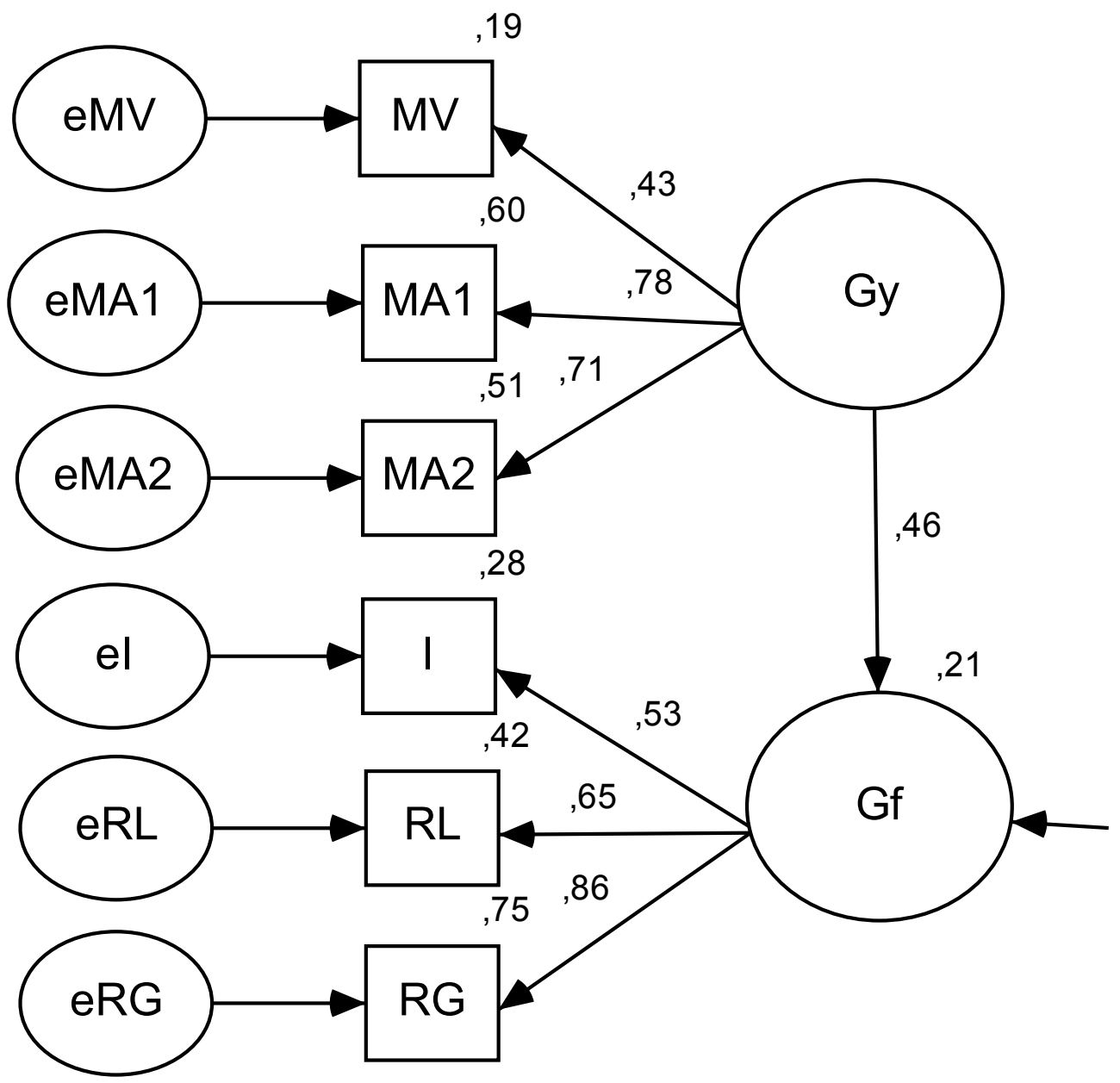

Figura 1. Representação Gráfica do Modelo dois.

Tabela 2

Peso das regressões não padronizadas do modelo dois, desvio padrão, Razão Crítica e Significância

Estatística das Regressões

\begin{tabular}{lccccrcc}
\hline & & & & & Razão & \\
\multicolumn{2}{r}{ Regressões do modelo } & Estimativa & $D P$ & crítica & $p$ \\
\hline Gf & $<---$ & Gy & 0,56 & 0,11 & 5,13 & $<0,001$ \\
MV & $<---$ & Gy & 0,34 & 0,06 & 5,49 & $<0,001$ \\
MA1 & $<---$ & Gy & 1,22 & 0,12 & 10,23 & $<0,001$ \\
MA2 & $<---$ & Gy & 1,00 & & & \\
I & $<---$ & Gf & 0,37 & 0,05 & 8,06 & $<0,001$ \\
RL & $<---$ & Gf & 0,79 & 0,08 & 9,68 & $<0,001$ \\
RG & $<---$ & Gf & 1,00 & & & \\
\hline
\end{tabular}




\section{Conclusão}

Pode-se sintetizar os principais resultados deste estudo da seguinte maneira. O Conjunto de Testes da Habilidade de Memória de Curto prazo (CTMC) mensura três habilidades cognitivas especializadas. São elas: a memória associativa verbal, a memória associativa numérica e a memória visual. Duas dessas habilidades correspondem a processos mais especializados da habilidade de memória associativa do primeiro estrato do modelo de Carroll (1993), enquanto a terceira habilidade, a memória visual, corresponde a uma habilidade do primeiro estrato. Essa evidência foi obtida através da análise fatorial exploratória. Analisando esse resultado, verifica-se uma corroboração dos argumentos presentes em Beier e Ackerman (2004), a respeito da importância dos conteúdos em testes de memória associativa. Há diferentes tipos de habilidades de memória associativa, de modo que a presença de dois testes distintos de memória associativa no CTMC traz um benefício à medida de Gsm e seus processos específicos

As evidências sugerem que o CTMC mensura a habilidade de memória de curto prazo (Gsm), além das habilidades específicas comentadas. Seus testes convergem para a medida de uma mesma habilidade-alvo e divergem da medida de outra habilidade cognitiva. Na medida em que os testes do CTMC possuem validade de conteúdo, conforme já argumentado, e convergem para um mesmo traço latente, distinto da medida de Gf, pode-se inferir que este traço seja Gsm. Evidentemente, uma evidência mais robusta para esta inferência será a occorrência de uma alta correlação entre a habilidade-alvo mensurada pelo CTMC e outros testes marcadores de Gsm validados para amostras brasileiras, de forma que a investigação da validade de critério deverá ser futuramente realizada para fins de averiguação da validade do CTMC, enquanto marcador de Gsm.

Do ponto de vista da validade preditiva, os resultados encontrados sugerem argumentar que Gsm medida pelo CTMC é uma habilidade de nível hierárquico diferente de Gf e aparentemente os modelos de processamento da informação que salientam o papel de base da memória estão corretos em seu postulado (Demetriou et al., 2002). Por sua vez, são necessários novos estudos que utilizem um número maior de testes com domínios variados, assim como amostras mais amplas e diversificadas.

Concluindo, a memória de curto prazo é um domínio investigado de forma vasta por uma série de tradições no campo da psicologia. Vários estudos da psicometria e da psicologia cognitiva têm se dedicado a estudar componentes e mecanismos da memória de curto prazo (Beaman, Neath, \& Surprenant, 2008; Farrell, 2008; Gabrieli, 1998; Galera \& Oliveira, 2004; Hollingworth, 2006; Jiang, Olson, \& Chun, 2000; Stuart \& Hulme, 2000; Tulving, 2002), assim como sua relação com outros processos cognitivos (Beier \& Ackerman, 2004). Esses estudos têm mostrado que o domínio da memória é complexo, formado por diversos componentes e relações intrincadas, e a memória de curto prazo é apenas um de seus diversos componentes.

O Conjunto de Testes da Habilidade de Memória (CTMC) é um conjunto que pretende mensurar a memória de curto prazo e contribuir junto aos estudos sobre memória. Pode-se dizer que, para a amostra estudada, o CTMC mostrou-se válido e confiável. Os seus testes convergem adequadamente para a mensuração da habilidade-alvo, assim como não participam da mensuração de outra habilidade cognitiva (Gf). Além disso, apresentam poder preditivo em relação à Gf, trazendo implicações para o campo dos estudos sobre a arquitetura cognitiva.

Com relação às suas limitações, é relevante que o CTMC venha a possuir novos testes, de outras habilidades do primeiro estrato de Carroll (1993) relacionadas à Gsm. Enquanto componente da BaFaCAlO, o CTMC parece ser um conjunto pertinente para a avaliação da inteligência, em seus diferentes níveis hierárquicos, em amostras brasileiras de ensino médio.

\section{Referências}

Alfonso, V. C., Flanagan, D. P., \& Radwan, S. (2005). The impact of the CattellHorn-Carroll theory on test development and interpretation of cognitive and academic abilities. In Dawn P. Flanagan \& Patti L. Harrison (Orgs.), Contemporary Intellectual Assessment: theories, tests, and issues (pp. 185202). New York: Guilford Press.

Beaman, C. P., Neath, I., \& Surprenant, A. M. (2008). Modeling distributions of immediate memory effects: no strategies needed? Journal of Experimental Psychology: Learning, Memory \& Cognition, 34(1), 219-229.

Beauducel, A. (2001). Problems with parallel analysis in data sets with oblique simple structure. Methods of Psychological Research Online, 6(2), 141-157.

Beier, M. E., \& Ackerman, P. L. (2004). A reappraisal of the relationship between span memory and intelligence via "best evidence synthesis". Intelligence, 32, 607-619.

Bickley, P. G., Keith, T. Z., \& Wolfle, L. M. (1995). The three-stratum theory of cognitive abilities: test of the structure of intelligence across the life span. Intelligence, 20, 309-328.

Byrne, B. M. (2001). Structural equation modeling with AMOS: basic concepts, applications, and programming. Mahwah, New Jersey: Lawrence Erlbaum Associates.

Capovilla, F. C., \& Capovilla, A. G. S. (2001). Compreendendo a natureza dos problemas de aquisição de leitura e escrita: mapeando o envolvimento de distúrbios cognitivos de discriminação fonológica, velocidade de processamento e memória fonológica. Cadernos de Psicopedagogia, 1(1), 14-37.

Carroll, J. B. (1993). Human cognitive abilities: a survey of factor analytic studies. New York: Cambridge University Press.

Carroll, J. B. (2003). The higher-stratum structure of cognitive abilities: current evidence supports $g$ and about ten broad factors. In H. Nyborg (Org.), The scientific study of general intelligence: tribute to Arthur R. Jensen (pp. 1-20). Elsevier Science/Pergamon Press.

Cole, J. C., \& Randall, M. K. (2003). Comparing the cognitive ability models of Spearman, Horn and Cattell, and Carroll. Journal of Psychoeducational Assessment, 21, 160-179.

Demetriou, A., Christou, C., Spanoudis, G., \& Platsidou, M. (2002). The development of mental processing: efficiency, working memory, and thinking. Monographs of the Society for Research in Child Development, 67(1), 1-171.

Ekstrom, R. B., French, J. W., Harman, H. H. \& Dirmen, D. (1976). Manual for kit of factor-referenced cognitive tests. Princeton: Educational Testing Service.

Farrell, S. (2008). Multiple roles for time in short-term memory: Evidence from serial recall of order and timing. Journal of Experimental Psychology: Learning, Memory \& Cognition, 34(1), 128-145.

Frazier, T. W., \& Youngstrom, E. A. (2007). Historical increase in the number of factors measured by commercial tests of cognitive ability: are we overfactoring? Intelligence, 35, 169-182.

Gabrieli, J. D. E. (1998). Cognitive neuroscience of human memory. Annual Review of Psychology, 49, 87-115. 
Galera, C. A., \& Oliveira, S. L. M. (2004). Quantidade e qualidade: duas abordagens da memória visuo-espacial. Paidéia, 14(27), 27-34.

Gomes, C. M. A. (2010). Estrutural fatorial da Bateria de Fatores Cognitivos de Alta-Ordem (BaFaCAlO). Avaliação Psicológica, 9(3), 449-459.

Gomes, C. M. A., \& Borges, O. N. (2009a). Qualidades psicométricas do Conjunto de Testes de Inteligência Fluida. Avaliação Psicológica, 8(1), 17-32.

Gomes, C. M. A., \& Borges, O. N. (2009b). Propriedades Psicométricas do Conjunto de Testes da Habilidade Visuo-Espacial. Psico-USF, 14(1), 19-34.

Guadagnoli, E., \& Velicer, W. F. (1988). Relation of sample size to the stability of component patterns. Psychological Bulletin, 103(2), 265-275.

Henson, R. K., \& Roberts, J. K. (2006). Use of exploratory factor analysis in published research: common errors and some comment on improved practice. Educational and Psychological Measurement, 66(3), 393-416.

Hollingworth, A. (2006). Scene and position specificity in visual memory for objects. Journal of Experimental Psychology: Learning, Memory \& Cognition, 32(1), 58-69.

Jiang, Y. H., Olson, I. R., \& Chun, M. M. (2000). Organization of visual shortterm memory. Journal of Experimental Psychology: Learning, Memory \& Cognition, 26(3), 683-702.

Johnson, W., \& Bouchard Jr., T. J. (2005a). Constructive replication of the visual perceptual-image rotation model in Thurstone's (1941) battery of 60 tests of mental ability. Intelligence, 33, 417-430.

Johnson, W., \& Bouchard Jr., T. J. (2005b). The structure of human intelligence: it is verbal, perceptual, and image rotation (VPR), not fluid and crystallized. Intelligence, 33, 393-416.

Johnson, W., te Nijenshuis, J., \& Bouchard Jr., T. J. (2007). Replication of the hierarchical visual-perceptual-image rotation model in de Wolff and Buiten's (1963) battery of 46 tests of mental ability. Intelligence, 35, 69-81.

Kranzler, J. H., Keith, T. Z., \& Flanagan, D. P. (2000). Independent examination fo the factor structure of the Cognitive Assessment System (CAS): further evidence challenging the construct validity of the CAS. Journal of Psychoeducational Assessment, 18, 143-159.

Marañon, R. C., \& Andrés-Pueyo, A. (2000). The study of human intelligence: a review at the turn of the millennium. Psychology in Spain, 4(1), 167-182.

McGrew, K.S., Keith, T.Z., Flanagan, D.P., \& Vanderwood, M. (1997). Beyond $\mathrm{g}$ : the impact of Gf-Gc specific cognitive ability research on the future use and interpretation of intelligence tests in the schools. School Psychology Review, 26, 189-201.

McGrew, K. S. \& Flanagan, D. P. (1998). The intelligence test desk reference (ITDR): Gf-Gc cross-battery assessment. Needham Heihts: Allyn \& Bacon.

Oberauer, K., Süß, H-M., Wilhelm, O., \& Wittman, W. W. (2003). The multiple faces of working memory: storage, processing, supervision, and coordination. Intelligence, 31, 167-193.

Primi, R. (2003). Inteligência: avanços nos modelos teóricos e nos instrumentos de medida. Avaliação Psicológica, 2(1), 67-77.

Roberts, R. D., Goff, G. N., Anjoul, F., Kyllonen, P. C., Pallier, G., \& Stankov, L. (2000). The Armed Services Vocational Aptitude Battery (ASVAB) little more than acculturated learning (Gc)!? Learning and Individual Differences, 12, 81-103

Rohde, T. E., \& Thompson, L. A. (2007). Predicting academic achievement with cognitive ability. Intelligence, 35, 83-92.

Rueda, F. J. M., Sisto, F. F., Cunha, C. A., \& Machado, F. (2007). Memória e atenção de escolares: um estudo comparativo. Encontro, 11, 66-78.

Rueda, F. J. M., Sisto, F. F., Cunha, C. A., Machado, F., Moraes Junior, R., Vitorino, F. C., \&

Souza, V. C. (2007). Evidências de validade para o Teste Pictórico de Memória: relação com a inteligência. Psicologia: Teoria e Prática, 9, 14-26.

Schelini, P. W., \& Wechsler, S. M. (2005). Bateria multidimensional de inteligência infantil: desenvolvimento de instrumento. Psico-USF, 10(2), 129-139.

Schelini, P. W., \& Wechsler, S. M. (2006). Estudo da estrutura fatorial da Bateria Multidimensional de Inteligência Infantil. Estudos de Psicologia (Campinas), 23, 105-112.

Spearrit, D. (1996). Carroll's model of cognitive abilities: educational implications. International Journal of Educational Research, 25(2), 107-198.

Stuart, G., \& Hulme, C. (2000). The effects of word co-occurrence on short-term memory: Associative links in long-term memory affect short-term memory performance. Journal of Experimental Psychology: Learning, Memory \& Cognition, 26(3), 796-802.

Tirre, W. C., \& Field, K. A. (2002). Structural models of abilities measured by the Ball Aptitude Battery. Educational and Psychological Measurement, $62,830-856$.

Tulving, E. (2002). Episodic memory: from mind to brain. Annual Review of Psychology, 53, 1-25.

Undheim, J. O., \& Gustafsson, J-E. (1987). The hierarchical organization of cognitive abilities: restoring general intelligence through the use of Linear Structural Relations (LISREL). Multivariate Behavioral Research, 22, 149-171.

Watkins, M. W., Wilson, S. M., Kotz, K. M., Carbone, M. C., \& Babula, T. (2006). Factor structure of the Wechsler Intelligence Scale for ChildrenFourth Edition among refereed students. Educational and Psychological Measurement, 66, 975-983.

Wechsler, S. M., \& Schelini, P. W. (2006). Bateria de habilidades cognitivas Woodcock-Johnson III: validade de construto. Psicologia: Teoria e Pesquisa, 22, 287-295.

Wechsler, S. M., Vendramini, C. M., \& Schelini, P. W. (2007). Adaptação brasileira dos testes verbais da bateria de habilidades cognitivas Woodcock-Johnson III. Interamerican Journal of Psychology, 41, 45-55.

Cristiano Mauro Assis Gomes, doutor em Educação pelo Programa de Pós-Graduação em Educação da Universidade Federal de Minas Gerais, é professor do Programa de Pós-Graduação em Psicologia e Programa de Pós-Graduação em Neurociências da Universidade Federal de Minas Gerais. Endereço para correspondência: Universidade Federal de Minas Gerais, Departamento de Psicologia. Av. Antônio Carlos 6627 Sala 4010, Pampulha, Belo Horizonte-MG. CEP: 31270-901. Telefone: (31)3409-6269. E-mail: cristianogomes@ufmg.br 